\title{
Hanbury-Brown-Twiss correlations and noise in the charge transfer statistics through a multiterminal Kondo dot
}

\author{
T. L. Schmidt ${ }^{1}$, A. Komnik ${ }^{2}$, and A. O. Gogolin, ${ }^{3}$ \\ 1 Physikalisches Institut, Albert-Ludwigs-Universität Freiburg, D-79104 Freiburg, Germany \\ ${ }^{2}$ Service de Physique Théorique, CEA Saclay, F-91191 Gif-sur-Yvette, France \\ ${ }^{3}$ Department of Mathematics, Imperial College London, \\ 180 Queen's Gate, London SW7 2AZ, United Kingdom
}

(Dated: June 29, 2018)

\begin{abstract}
We analyze the full counting statistics of charge transfer through a quantum dot in the Kondo regime, when coupled to an arbitrary number of terminals $N$. At the unitary Kondo fixed point and for $N>2$ we recover distinct anticorrelations of currents in concurring transport channels, which are related to the fermionic Hanbury Brown and Twiss (HBT) antibunching. This effect weakens as one moves away from the fixed point. Furthermore, we identify a special class of current correlations that are due entirely to the virtual polarization of the Kondo singlet. These can be used for extracting information on the parameters of the underlying Fermi-liquid model.
\end{abstract}

PACS numbers: 72.10.Fk, 73.63.-b, 73.23.-b, 72.15.Qm

Since its discovery the Kondo effect remains one of the most active fields in condensed matter physics [1]. The last decade witnessed a very important achievement from the experimental point of view: the creation of a fully adjustable artificial Kondo impurity [2, 3, 4]. This enables one, in principle, to measure almost any transport property of the system in a wide parameter range. One impressive example is the Kondo resonance splitting, which occurs under nonequilibrium conditions when the impurity is simultaneously coupled to two terminals held at different chemical potentials 5, 6, 7]. It turned out that the best way to explore this effect is to introduce a third electrode and measure the tunnelling density of states $[8,9]$. This fact triggered a number of different studies of the Kondo effect in multiterminal geometries 10, 11, 12, 13.

Apart from peak splitting measurements, artificial Kondo impurities are also interesting for another reason. As was pointed out in early works on transport in multiterminal systems, they possess some unique transport properties due to a possibility of having multiple concurrent transport channels. In particular, the fermionic nature of charge carriers leads to antibunching of individual transport events, also called fermionic Hanbury Brown and Twiss (HBT) correlations 14, 15]. This reveals itself as a negative sign of the current-current correlation function between concurring transport channels. For the simplest three-terminal geometry this effect has been confirmed experimentally in [16, 17]. Thus far little has been done to investigate these effects in the Kondo setup. The most advanced work is [12], where the current-current correlations have been analysed for the three-terminal geometry using the slave-boson approximation. To the best of our knowledge, nothing is known about the correlations of higher orders or close to the fixed point. With this Letter we would like to close this gap by addressing the full counting statistics (FCS) of such setups near the unitary limit.
We start with the following Hamiltonian,

$$
H=\sum_{j=1}^{N} H_{0}\left[\psi_{j \sigma}\right]+J \mathbf{S} \sum_{i, j=1}^{N} \sum_{\sigma \sigma^{\prime}} \psi_{i \sigma}^{\dagger}(0) \boldsymbol{\tau}_{\sigma \sigma^{\prime}} \psi_{j \sigma^{\prime}}(0)
$$$$
-h S^{z},(1)
$$

where the first sum describes the dynamics of the electrons $H_{0}\left[\psi_{j \sigma}\right]=\sum_{p \sigma j}\left(\epsilon_{p}-V_{j}\right) \psi_{p \sigma j}^{\dagger} \psi_{p \sigma j}$ in the noninteracting leads (We set $e=\hbar=k_{B}=1$ throughout. The conductance quantum then becomes $G_{0}=2 e^{2} / h=1 / \pi$ ). Here $\psi_{p \sigma j}$ is the annihilation operator for an electron with momentum $p$, spin $\sigma$ in the $j$ th terminal held at chemical potential $V_{j}$. The second part represents the coupling of the local spin degrees of freedom in the leads to the impurity spin $\mathbf{S}: J$ is the respective coupling constant, $\boldsymbol{\tau}$ denotes the set of Pauli matrices, $h=\mu_{B} g B$ is the product of Bohr's magneton $\mu_{B}$, of the gyromagnetic ratio $g$ and the magnetic field $B$ applied to the impurity. Via simple unitary transformation to a set of new fermions $c_{j \sigma}$ this Hamiltonian can be mapped onto the single channel Kondo model, where only one of the new fields, $c_{1 \sigma}$, remains coupled to the impurity spin:

$$
c_{1 \sigma}=\frac{1}{\sqrt{N}} \sum_{j=1}^{N} \psi_{j \sigma} .
$$

In the low energy sector, well below the Kondo temperature $T_{K}$, this fermion degree of freedom is hybridised with the impurity spin into a singlet state. The true fixed point of the system is just an $N$-leg resonant model. The effective fixed-point Hamiltonian is simply $H_{0}$ for $c_{1 \sigma}$, supplemented by voltage terms. The best possible transparency is then realized by the following scattering matrix $\psi_{j}\left(0^{+}\right)=s_{j k} \psi_{k}\left(0^{-}\right)\left(0^{ \pm}\right.$denote in- and out-going plane waves), with [18]

$$
s_{j j}=(N-2) / N, s_{j k}=-2 / N \text { for } j \neq k .
$$

The central object in FCS calculations is the cumulant generating function (CGF) $\chi(\boldsymbol{\lambda})=\sum_{\boldsymbol{Q}} e^{i \mathbf{Q} \boldsymbol{\lambda}} P(\mathbf{Q})$ 
[19], where $\boldsymbol{\lambda}=\left(\lambda_{1}, \ldots, \lambda_{N}\right)$ are the measuring fields and $P(\mathbf{Q})$ is the probability that charges $\mathbf{Q}=\left(Q_{1}, \ldots, Q_{N}\right)$ are transferred across the respective channels during the waiting time $\mathcal{T}$. The cumulants (irreducible moments) are then found from the respective derivatives of $\chi(\boldsymbol{\lambda})$. With the knowledge of the scattering matrix (no interactions) the CGF is given by the multiterminal version of the Levitov-Lesovik formula [20]:

$$
\ln \chi_{0}(\boldsymbol{\lambda})=\frac{\mathcal{T}}{\pi} \int d \omega \ln \operatorname{det}\left[1+\hat{f}(\omega)\left(\hat{s}^{\dagger} \widetilde{s}-1\right)\right],
$$

where $\widetilde{s}_{m n}=e^{i\left(\lambda_{n}-\lambda_{m}\right)} s_{m n}$ and the diagonal matrix $\hat{f}(\omega)=\operatorname{diag}\left(n_{1}, n_{2}, \ldots, n_{N}\right)$ contains the Fermi distribution functions of individual terminals $n_{i}$. One then easily verifies that the fixed point $\mathrm{CGF}$ is given by:

$$
\begin{aligned}
\ln \chi_{0}(\boldsymbol{\lambda}) & =\frac{\mathcal{T}}{\pi} \\
\times \quad \int d \omega \ln & \left\{1+\left(\frac{2}{N}\right)^{2} \sum_{i \neq j}\left[e^{i\left(\lambda_{j}-\lambda_{i}\right)}-1\right] n_{i}\left(1-n_{j}\right)\right\} .
\end{aligned}
$$

In the particular case of zero temperature and three terminals such that $V_{1}>V_{2}>V_{3}$ the above formula becomes

$$
\begin{aligned}
& \ln \chi_{0}(\boldsymbol{\lambda})= \\
& \quad \frac{\mathcal{T}}{\pi}\left\{\left(V_{1}-V_{2}\right) \ln \left[1+\frac{4}{9}\left(e^{i\left(\lambda_{1}-\lambda_{3}\right)}+e^{i\left(\lambda_{1}-\lambda_{2}\right)}-2\right)\right]\right. \\
& \left.+\left(V_{2}-V_{3}\right) \ln \left[1+\frac{4}{9}\left(e^{i\left(\lambda_{1}-\lambda_{3}\right)}+e^{i\left(\lambda_{2}-\lambda_{3}\right)}-2\right)\right]\right\} .
\end{aligned}
$$

Here one recognises that the current pairs $I_{1 \rightarrow 3} \& I_{1 \rightarrow 2}$ and $I_{1 \rightarrow 3} \quad \& \quad I_{2 \rightarrow 3}$ are mutually correlated, the corresponding fluctuations of charge differences in the respective channels being [14]

$$
\begin{aligned}
\left\langle\delta\left(Q_{1}-Q_{2}\right) \delta\left(Q_{1}-Q_{3}\right)\right\rangle & =-\frac{16}{81 \pi} \mathcal{T}\left(V_{1}-V_{2}\right), \\
\left\langle\delta\left(Q_{1}-Q_{3}\right) \delta\left(Q_{2}-Q_{3}\right)\right\rangle & =-\frac{16}{81 \pi} \mathcal{T}\left(V_{2}-V_{3}\right) .
\end{aligned}
$$

The negative sign is the signature of fermion antibunching, or HBT type correlations. On the other hand, the pair $I_{1 \rightarrow 2} \& I_{2 \rightarrow 3}$ is not entangled in any way, as those transport channels are not concurring. This is true only at $T=0$, of course, its leading temperature dependence being

$$
\left\langle\delta\left(Q_{1}-Q_{2}\right) \delta\left(Q_{2}-Q_{3}\right)\right\rangle=-\frac{16}{81 \pi} \mathcal{T} T .
$$

for $T \ll\left|\min \left(V_{i}-V_{j}\right)\right|, i \neq j$. In contrast to cross correlations, the autocorrelation functions are positive, e. g. one finds for the current noise between the channels 2 and 3

$$
\left\langle\delta^{2}\left(Q_{2}-Q_{3}\right)\right\rangle=\frac{20}{81} \frac{\mathcal{T}}{\pi}\left(V_{2}-V_{3}\right) .
$$

For $V_{2}=V_{3}$ the leading temperature dependence of this correlation is again linear,

$$
\left\langle\delta^{2}\left(Q_{2}-Q_{3}\right)\right\rangle=\frac{8}{9 \pi} \mathcal{T} T
$$

One can show that these cumulants vanish whenever $V_{2}=V_{3}$ and $T=0$ even away from resonance and for a lead- asymmetric junction.

The departure from the Kondo fixed point is described by the Nozières Fermi-liquid theory [21]. The effective Hamiltonian contains then in addition to the free part a scattering term

$$
H_{\mathrm{Sc}}=\frac{\alpha}{2 \pi \nu T_{K}} \sum_{p p^{\prime} \sigma}\left(\epsilon_{p}+\epsilon_{p}^{\prime}\right) c_{1 p \sigma}^{\dagger} c_{1 p^{\prime} \sigma}
$$

with a dimensionless amplitude $\alpha$ and local density of states at the Fermi energy of the leads $\nu$ (assumed for simplicity to be equal for all terminals), and an interaction:

$$
H_{\mathrm{int}}=\frac{\phi}{\pi \nu^{2} T_{K}}\left(c_{1 \uparrow}^{\dagger} c_{1 \uparrow}-n_{0}\right)\left(c_{1 \downarrow}^{\dagger} c_{1 \downarrow}-n_{0}\right),
$$

with an amplitude $\phi$. Here $c_{1 \sigma}=\sum_{p} c_{1 p \sigma}$ and $n_{0}$ stands for the particle density in the zero field and accounts for the background ion charge. The constant $\alpha$ is responsible for the impurity specific heat whereas the sum $\alpha+\phi$ governs the magnetic susceptibility of the system. In the actual Kondo model the Fermi-liquid relation $\alpha=\phi=1$ holds 21].

The standard way to calculate the FCS is to introduce time-dependent counting fields $\boldsymbol{\lambda}$ (nonzero only during the waiting time $\mathcal{T}$ ) coupled to charge currents [19, 22]. In the alternative procedure such terms can be gauged away by a canonical transformation [23], which is easily generalized to the multi-terminal geometry. This transformation is performed at the expense of having $\lambda$ dependent exponentials embedded into the interaction parts of the Hamiltonian via $H_{\mathrm{Sc}}\left[c_{1}\right]+H_{\text {int }}\left[c_{1}\right] \rightarrow H_{\lambda}\left[c_{1}\right]$. The $\mathrm{CGF}$ is then calculated according to the prescription [24],

$$
\begin{aligned}
\chi(\boldsymbol{\lambda}) & =\chi_{0}(\boldsymbol{\lambda}) \chi_{1}(\boldsymbol{\lambda}) \\
& =\chi_{0}(\boldsymbol{\lambda})\left\langle T_{C} \exp \left[-i \int_{C} d t H_{\lambda}(t)\right]\right\rangle,
\end{aligned}
$$

where $C$ denotes the Keldysh contour and $T_{C}$ is the time ordering operator on it [25]. The evaluation of $\chi_{1}(\boldsymbol{\lambda})$ can now be accomplished via the standard linked cluster expansion in the couplings $\alpha, \phi$ using the Green's functions of the $\boldsymbol{\lambda}$-rotated $c_{1 \sigma}$ operator:

$$
\begin{gathered}
\hat{g}_{\lambda}(\sigma, p, \omega)=i \pi \frac{2}{N} \delta\left(\varepsilon_{p}-\omega\right) \sum_{j=1}^{N}\left\{\left[n_{j \sigma}(\omega)-1 / 2\right] \boldsymbol{\tau}_{0}\right. \\
\left.+e^{-i \lambda_{j} / 2} n_{j \sigma}(\omega) \boldsymbol{\tau}_{+}-\left[1-n_{j \sigma}(\omega)\right] e^{i \lambda_{j} / 2} \boldsymbol{\tau}_{-}\right\}
\end{gathered}
$$

where $\boldsymbol{\tau}_{ \pm}$are the standard Pauli matrices, $\boldsymbol{\tau}_{0}$ is the unity matrix and $n_{j \sigma}$ stand for the Fermi distribution function in the respective terminal. 
To the lowest (second) order there are three different contributions: the ones proportional to $\alpha^{2}, \phi^{2}$, and $\alpha \phi$. The latter cross term is nonzero only in finite field. The $\alpha^{2}$ part emerges as

$$
\begin{array}{r}
\delta_{\alpha} \ln \chi_{1}=\left(\frac{2}{N}\right)^{2} \frac{\alpha^{2} \mathcal{T}}{2 \pi T_{K}^{2}} \sum_{m \neq n}^{N} \sum_{\sigma}\left[e^{-i\left(\lambda_{m}-\lambda_{n}\right) / 2}-1\right] \\
\times I_{1}\left(V_{m}+h \sigma, V_{n}+h \sigma\right),
\end{array}
$$

where we define the function

$$
I_{1}\left(V_{1}, V_{2}\right)=\left(V_{1}-V_{2}\right) \frac{(\pi T)^{2}+V_{1}^{2}+V_{2}^{2}+V_{1} V_{2}}{3\left[1-e^{-\left(V_{1}-V_{2}\right) / T}\right]}
$$

Evaluation of the interaction $\phi^{2}$ correction results in two different contributions. The first one is field independent and for the case of $N=3$ terminals (a general result for arbitrary $N$ exists but is quite lengthy) is found to be

$$
\begin{aligned}
& \delta_{\phi}^{\prime} \ln \chi_{1}=\frac{1}{3^{4}} \frac{\phi^{2} \mathcal{T}}{\pi T_{K}^{2}}\left\{\sum_{m \neq n}^{3}\left[8 e^{-i\left(\lambda_{m}-\lambda_{n}\right) / 2} I_{2}\left(V_{m}-V_{n}\right)+e^{-i\left(\lambda_{m}-\lambda_{n}\right)} I_{2}\left[2\left(V_{m}-V_{n}\right)\right]\right]\right. \\
& \left.+2 \sum_{m=1}^{3}\left[\exp \left(-i \lambda_{m}+i / 2 \sum_{n \neq m}^{3} \lambda_{n}\right) I_{2}\left(2 V_{m}-\sum_{n \neq m}^{3} V_{n}\right)+\exp \left(i \lambda_{m}-i / 2 \sum_{n \neq m}^{3} \lambda_{n}\right) I_{2}\left(-2 V_{m}+\sum_{n \neq m}^{3} V_{n}\right)\right]\right\}
\end{aligned}
$$

where we have defined the function

$$
I_{2}(V)=\frac{V}{3} \frac{4(\pi T)^{2}+V^{2}}{1-e^{-V / T}} .
$$

On the other hand, for the field-dependent correction we obtain

$$
\begin{array}{r}
\delta_{\phi}^{\prime \prime} \ln \chi_{1}=\left(\frac{2}{N}\right)^{2} \frac{\phi^{2} h^{2} \mathcal{T}}{\pi T_{K}^{2}} \sum_{m \neq n}^{N}\left[e^{-i\left(\lambda_{m}-\lambda_{n}\right) / 2}-1\right] \\
\times \frac{V_{m}-V_{n}}{1-e^{-\left(V_{m}-V_{n}\right) / T}} .
\end{array}
$$

Finally, the $\alpha \phi$ cross term, which is also nonzero in a finite field only, is given by

$$
\begin{aligned}
\delta_{\alpha \phi} \ln \chi_{1} & =\left(\frac{2}{N}\right)^{2} \frac{2 \alpha \phi h^{2} \mathcal{T}}{\pi T_{K}^{2}} \sum_{m \neq n}^{N}\left[e^{-i\left(\lambda_{m}-\lambda_{n}\right) / 2}-1\right] \\
& \times \frac{V_{m}-V_{n}}{1-e^{-\left(V_{m}-V_{n}\right) / T}} .
\end{aligned}
$$

The full FCS contains all transport characteristics of the system. Here we would like to concentrate on two rather interesting issues. The first question we want to address is to what extent are the HBT type correlations affected away from the fixed point. In order to discuss this we again use the convention $V_{1}>V_{2}=V_{3}$. Then the transport channels $1 \rightarrow 2,1 \rightarrow 3$ become concurrent. The only term, still containing both differences of counting fields $\lambda_{1}-\lambda_{2,3}$, can be found in Eq. (15). The respective correction to (7) at $T=0$ is given by

$$
\left\langle\delta\left(Q_{1}-Q_{2}\right) \delta\left(Q_{1}-Q_{3}\right)\right\rangle=\frac{4}{3^{5}} \frac{\phi^{2} \mathcal{T}}{\pi T_{K}^{2}}\left(V_{1}-V_{2}\right)^{3} .
$$

We see that the residual Kondo interactions lead to a weakening of the HBT signature. The remarkable fact is that this correction only depends on $\phi$ as well as its cubic voltage behaviour (in contrast to the linear one of the conventional HBT) offers an opportunity to extract this effective parameter from the experimental data. However, the original HBT background might complicate the measurements.

Luckily, this difficulty can be avoided by measurement of different correlation functions. As we have already mentioned above, at the fixed point there is no entanglement between nonconcurrent channels. This is different in the Kondo system. For instance, under the above conditions there is a residual correlation between the particle numbers in channels 1 and 3, which is absent at the true unitary limit. Using the result (15) we immediately verify that

$$
\left\langle\delta\left(Q_{1}-Q_{2}\right) \delta\left(Q_{2}-Q_{3}\right)\right\rangle=-\frac{\phi^{2} \mathcal{T}}{2 \cdot 3^{5} \pi T_{K}^{2}}\left(V_{1}-V_{2}\right)^{3}
$$

Comparing this equation with the temperature dependence of the primary contribution (8) we see that the interplay of nonequilibrium and polarization of the Kondo singlet amounts to residual correlations at $T=0$. Similar fate meets the autocorrelation (noise) of the $I_{2 \rightarrow 3}$ current. While it is zero in the unitary limit, Eq. (9) for $V_{2}=V_{3}$, it acquires a finite value away from the fixed point,

$$
\left\langle\delta^{2}\left(Q_{2}-Q_{3}\right)\right\rangle=\frac{\phi^{2} \mathcal{T}}{3^{5} \pi T_{K}^{2}}\left(V_{1}-V_{2}\right)^{3} .
$$


Since in this limit there is no background from the leading order, we expect that these types of correlation would be a good means to access the parameter $\phi / T_{K}$ in future experiments. Interestingly, the finite magnetic field does not affect these conclusions.

As the system under consideration is a Fermi liquid it is natural to ask whether there is a possibility to use this fact in order to derive a universal CGF valid also beyond the leading corrections to the unitary limit. Indeed, in the case of two terminals Oguri succeeded in deriving an expression for the current for a more general Anderson impurity model for arbitrary on-site repulsion $U$ for small voltages [26]. In [25] a related formula for a CGF has been proposed, valid around the unitary limit as well as for small $U$ [23]. It turns out that a similar program can be performed in the present multiterminal case. Indeed, the cross-voltage derivatives, $\partial^{2} / \partial V_{1} \partial V_{2}$, of the bare Green's functions vanish. Therefore the method of Ref. 26] applies directly. For the relative currents [the actual currents in the respective channels being given by $\left.I_{i}=(1 / N) \sum_{j} I_{i \rightarrow j}\right]$ we find

$$
\begin{array}{r}
I_{i j}=4 \frac{V_{i}-V_{j}}{\pi N}\left[1-\frac{2 \chi_{e}^{2}+\chi_{o}^{2}}{6 \Gamma_{N}^{2}}\left(V_{i}^{2}+V_{i} V_{j}+V_{j}^{2}\right)\right. \\
\left.-\frac{3 \chi_{o}^{2}}{2 N \Gamma_{N}^{2}} \sum_{i} V_{i}^{2}+\ldots\right]
\end{array}
$$

where $\Gamma_{N}=N \Gamma=N \pi \rho_{0} \gamma^{2}, \gamma$ is the tunnelling amplitude coupling the Anderson impurity level to the terminals with the local tunnelling density of states $\rho_{0}$. The objects $\chi_{e, o}$ are the even/odd susceptibilities which are correlations of impurity population probabilities with the same/different spin orientations, respectively. This result is valid for small voltages $V_{i} \ll \Gamma_{N}$ and is exact in interaction as the $U$ dependence is now contained in $\chi_{e, o}$. These are essentially equilibrium quantities and can be found with other techniques, e. g. by Bethe ansatz. In the unitary limit the identification of coefficients is very simple: $\chi_{e}=\Gamma_{N} \alpha / T_{K}$ and $\chi_{o}=\Gamma_{N} \phi / T_{K}$. After that substitution one then recovers the results found from the CGF (13). We expect that also in Eq. (20) and similar formulas the parameter $\phi$ can be substituted by the exact susceptibility $\chi_{o}$, even though we do not have a formal proof of this statement.

To summarize, we report analytical results for the charge transfer statistics for the Kondo impurity coupled to an arbitrary number of terminals. In the Kondo limit, when bias voltage, magnetic field and temperature are all smaller than the Kondo temperature, we use a generalization of the Nozières Fermi-liquid theory to derive a perturbative expansion for the FCS in leading irrelevant operators. At the true fixed point we recover with our method the Hanbury Brown and Twiss anticorrelations between concurrent transport channels. While these turn out to weaken away from the unitary limit, new correlations emerge due to the virtual polarization of the Kondo singlet. The most promising of these is the low- $T$ noise in the $2-3$ channel of the $N=3$ junction at $V_{1}>V_{2}=V_{3}$. Measurements of the latter would enable one to directly access the effective parameters of the Fermi liquid model in experiments similar to those of [16, 17].

The authors participate in the European network DIENOW. TLS and AK acknowledge the support by the DFG. AK is supported by the Feodor Lynen program of the Alexander von Humboldt foundation.
[1] A. C. Hewson, The Kondo Problem to Heavy Fermions (Cambridge University Press, Cambridge, 1997).

[2] D. Goldhaber-Gordon, H. Shtrikman, D. Mahalu, D. Abusch-Magder, U. Meirav, and M. A. Kastner, Nature 391, 156 (1998).

[3] S. M. Cronenwett, T. H. Oosterkamp, and L. P. Kouwenhoven, Science 281, 540 (1998).

[4] J. Schmid, J. Weis, K. Eberl, and K. von Klitzing, Physica B 256-258, 182 (1998).

[5] Y. Meir, N. S. Wingreen, and P. A. Lee, Phys. Rev. Lett. 70, 2601 (1993).

[6] J. König, J. Schmid, H. Schoeller, and G. Schön, Phys. Rev. B 54, 16820 (1996).

[7] A. Rosch, J. Kroha, and P. Wölfle, Phys. Rev. Lett. 87, 156802 (2001).

[8] Q.-F. Sun and H. Guo, Phys. Rev. B 64, 153306 (2001).

[9] E. Lebanon and A. Schiller, Phys. Rev. B 65, 035308 (2001).

[10] S. De Franceschi, R. Hanson, W. G. van der Wiel, J. M. Elzerman, J. J. Wijpkema, T. Fujisawa, S. Tarucha, and L. P. Kouwenhoven, Phys. Rev. Lett. 89, 156801 (2002).

[11] R. Leturcq, L. Schmid, K. Ensslin, Y. Meir, D. C. Driscoll, and A. C. Gossard, Phys. Rev. Lett. 95, 126603
(2005).

[12] D. Sanchez and R. Lopez, Phys. Rev. B 71, 035315 (2005).

[13] N. Shah and A. Rosch, Phys. Rev. B 73, 081309 (2006).

[14] M. Büttiker, Phys. Rev. B 46, 12485 (1992).

[15] T. Martin, in Les Houches Session LXXXI, edited by H. Bouchiat et al. (Elsevier, 2005).

[16] W. D. Oliver, J. Kim, R. C. Liu, and Y. Yamamoto, Science 284, 299 (1999).

[17] M. Henny, S. Oberholzer, C. Strunk, T. Heinzel, K. Ensslin, M. Holland, and C. Schönenberger, Science 284, 296 (1999).

[18] C. Nayak, M. P. A. Fisher, A. W. W. Ludwig, and H. H. Lin, Phys. Rev. B 59, 15694 (1999).

[19] L. S. Levitov, W. W. Lee, and G. B. Lesovik, Journ. Math. Phys. 37, 4845 (1996).

[20] L. S. Levitov and G. B. Lesovik, JETP Lett. 58, 230 (1993).

[21] P. Nozières, J. Low Temp. Phys. 17, 31 (1974).

[22] Yu. V. Nazarov, Ann. Phys. (Leipzig) 8 (SI-193), 507 (1999).

[23] A. O. Gogolin and A. Komnik, Phys. Rev. Lett. 97, 016602 (2006). 
[24] L. S. Levitov and M. Reznikov, Phys. Rev. B 70, 115305 (2004).

[25] A. O. Gogolin and A. Komnik, Phys. Rev. B 73, 195301

(2006).

[26] A. Oguri, Phys. Rev. B 64, 153305 (2001). 